$\mathrm{PM} / 96-25$

FNT/T-96/18

September 1996

\title{
Observability at LEP2 hadronic channels of a Z' with small lepton couplings
}

\author{
G. Montagna ${ }^{a, b}$, F. Piccinini ${ }^{b, a}$ \\ J. Layssac ${ }^{c}$, F.M. Renard ${ }^{c}$ \\ C. Verzegnassi ${ }^{d}$ \\ ${ }^{a}$ Dipartimento di Fisica Nucleare e Teorica, Università di Pavia \\ Via A.Bassi 6, 27100, Pavia, Italy. \\ ${ }^{b}$ INFN, Sezione di Pavia, Via A.Bassi 6, 27100, Pavia, Italy. \\ ${ }^{c}$ Physique Mathématique et Théorique, UPRES-A 5032, \\ Université de Montpellier II, F-34095 Montpellier Cedex 5. \\ ${ }^{d}$ Dipartimento di Fisica, Università di Lecce \\ CP193 Via Arnesano, I-73100 Lecce, \\ and INFN, Sezione di Lecce, Italy.
}

\begin{abstract}
We consider the effects of a number of models with one extra $Z$, with enhanced couplings to quarks, in the final hadronic channels at LEP2. We show that, for a number of representative cases, visible effects could be produced even for very low values of the lepton couplings, much smaller than the existing LEP1/SLC and the future LEP2 (lepton channel) bounds.
\end{abstract}




\section{Introduction}

It has been recently suggested [1], [2] that a model with one extra $Z \equiv Z^{\prime}$ with enhanced, family independent couplings to quarks, would explain in a simple way possible departures from the SM predictions for the $Z$ couplings to heavy quarks [3] and, at the same time, a possible excess of dijet events at CDF [4]. For non vanishing values of its charged lepton couplings, it would be possible to see the effects of such a $Z^{\prime}$ ("hadrophilic" in the notation of Ref. [1]) in the final hadronic channels at LEP2 [1] under the expected experimental accuracy for the relevant observables [5].

Following the original proposal of Refs. [1], [2], several papers have appeared [6], [7] that investigate the possibility of finding a realistic theoretical model where this special $Z^{\prime}$ can be accomodated without introducing unwanted anomalies. In the original proposal of Ref. [6], this goal was achieved assuming that the couplings of the $Z^{\prime}$ with leptons were ("approximately") vanishing. This generated the word "leptophobic" $Z$ ', the "leptophobia" syndrome being substantially supported by the phenomenological evidence that leptonic widths and asymmetries are in very good agreement with the SM predictions and do not necessitate, in principle, any correction of New Physics type.

A rather fundamental detail that we want to stress at this point is that, from an experimental point of view, there is a dramatic difference between a $Z^{\prime}$ with "approximately" vanishing lepton couplings and one with exactly vanishing lepton couplings. The latter one, strictly speaking, could not be revealed by the conventional techniques, based on searches of virtual $Z^{\prime}$ effects, at future $e^{+} e^{-}$colliders [8], [9], so that LEP2 and NLC would be "blind" to such an effect, even for very low values of the $Z^{\prime}$ mass. On the contrary, a $Z^{\prime}$ with "small" lepton couplings might compensate this kind of weakness with a suitable enhancement of its quark couplings. In this case, the final hadronic channels at LEP2 and NLC might be able to evidentiate the hadrophilic $Z^{\prime}$ virtual effect in the conventional way, i.e. via small deviations in the hadronic cross section and/or asymmetries, even for substantial (in the LEP2 case, of about $1 \mathrm{TeV}$ ) values of the $Z^{\prime}$ mass.

Since the point that we have just mentioned seems to us worth of being investigated in more detail, we have decided to devote this paper to a discussion of the "minimal" size of the lepton couplings that would still make a hadrophilic $Z^{\prime}$ visible at LEP2 .

The outline of the paper is as follows. We describe in Section 2 a theoretical model where one $Z^{\prime}$ exists, with particular emphasis on its phenomenological inputs and constraints. In section 3 we present the implementation of the QED radiative corrections in the calculation of the $Z^{\prime}$ effects on the hadronic observables. The discussion of the numerical results is given in Section 4 and the main conclusions are summarized in Section 5.

\footnotetext{
${ }^{1}$ and, therefore, not "LEP2-phobic", but rather, in a generalized notation, "LEP2-philic"
} 


\section{Phenomenological constraints and theoretical model}

Since we shall assume that $Z^{\prime}$ quark couplings are "large", we shall introduce and use from now on four parameters that are indicative of this relative enhancement, more precisely the ratios

$$
\xi_{V f} \equiv \frac{g_{V f}^{\prime}}{g_{V f}} \quad ; \quad \xi_{A f} \equiv \frac{g_{A f}^{\prime}}{g_{A f}}
$$

where $g_{V, A f}(f=u, d)$ are the usual $Z f \bar{f}$ couplings, $g_{V f}=I_{3 L}^{f}-2 s_{\text {eff }}^{2} Q^{f}$, where $s_{e f f}^{2}=$ 0.232 and $g_{A f}=I_{3 L}^{f}$. The $Z^{\prime}$ effect in the hadronic channels at LEP2 will depend on six effective parameters, those of eq. (1) and the conventionally defined $Z^{\prime}$ lepton couplings $g_{V l}^{\prime}$ and $g_{A l}^{\prime}$ (we follow the convention of Ref. [1]), each one divided by $\sqrt{M_{Z^{\prime}}^{2}-q^{2}}$, where $q^{2}$ is the total c.m. squared energy. We shall assume at this stage that the $Z^{\prime}$ couplings are family independent.

To give a hint of the practical situation, we now write the approximate expressions (computed at the lowest order "effective" Born level) of the relative shifts from the SM predictions (due to a general $Z^{\prime}$ ) of the three experimental hadronic quantities that will be realistically measured at LEP2, i.e. the total hadronic cross section $\sigma_{5}$ and the $b \bar{b}$ cross section and forward-backward asymmetry $\sigma_{b}, A_{F B, b}$. For these observables it is not difficult to derive the following expressions for the aforementioned shifts, that read, in the chosen configuration $\sqrt{q^{2}}=175 \mathrm{GeV}$ (where we shall work from now on) 10 and for large $M_{Z^{\prime}}$ values, $M_{Z^{\prime}}^{2} \gg M_{Z}^{2}$ (chosen in this illustrative example for pure simplicity reasons):

$$
\begin{gathered}
\frac{\delta \sigma_{5}^{\left(Z^{\prime}\right)}}{\sigma_{5}} \simeq\left[1.4 \times 10^{-2}\right]\left(\frac{1 T e V}{M_{Z}^{\prime}}\right)^{2}\left\{\begin{array}{r}
\left\{g_{V l}^{\prime}\left[0.67 \xi_{V u}+0.83 \xi_{V d}+0.232 \xi_{A u}+0.41 \xi_{A d}\right]\right. \\
\left.+g_{A l}^{\prime}\left[1.64 \xi_{A d}+1,10 \xi_{A u}+0.17 \xi_{V u}+0.84 \xi_{V d}\right]\right\}
\end{array}\right. \\
\frac{\delta \sigma_{b}^{\left(Z^{\prime}\right)}}{\sigma_{b}} \simeq\left[5 \times 10^{-2}\right]\left(\frac{1 T e V}{M_{Z}^{\prime}}\right)^{2}\left\{g_{V l}^{\prime}\left[0.50 \xi_{V d}+0.19 \xi_{A d}\right]+g_{A l}^{\prime}\left[0.96 \xi_{A d}+0.46 \xi_{V d}\right]\right\} \\
\frac{\delta A_{F B, b}^{\left(Z^{\prime}\right)} \simeq\left[10 \times 10^{-2}\right]\left(\frac{1 T e V}{M_{F B, b}^{\prime}}\right)^{2}\left\{g_{V l}^{\prime}\left[0.21 \xi_{V d}+0.40 \xi_{A d}\right]+g_{A l}^{\prime}\left[-0.23 \xi_{V d}-0.05 \xi_{A d}\right]\right\}}{A_{F B}}
\end{gathered}
$$

Eqs. (2) -(14) can be easily derived within a special theoretical framework, denoted " $Z$ peak subtracted" representation of four-fermion processes [11, [12]. In fact, the complete relevant expression for general $q^{2}$ and $M_{Z^{\prime}}$ values can be found in Ref. [12], and we shall not insist here on their derivation, particularly since a more complete evaluation of the effects, that takes the dangerous QED radiation properly into account, will be performed 
in this paper. The reason why we showed these approximate expressions is that from their inspection a certain number of general conclusions can be already drawn. In particular:

I) the numerical square brackets that appear as first terms of the r.h.s. of eqs. (2)(4) are the expected experimental accuracies for the various quantities (two standard deviations) at LEP2. In order to make a visible effect, all the quantities in the three curly brackets must be (at least) of order one. For values of $\left|\xi_{i}\right|$ reasonably larger than one (say, of about $\sim 3$ to $\sim 4)$ one sees that e.g. values of $g_{V l}^{\prime}=g_{A l}^{\prime} \simeq \pm 0.1\left(M_{Z^{\prime}} / 1 \mathrm{TeV}\right)$ would produce a visible effect in $\sigma_{5}$ and also in $\sigma_{b}$. These indicative results will be substantially confirmed by the more accurate determination that we shall perform.

II) The three experimental observables of eqs. (2)-(4) clearly exhibit a different dependence on the four quark ratios $\xi_{i}$. This means that a certain non trivial correlation will exist between the three " $Z$ ' shifts" that might be useful to differentiate a model with a $Z^{\prime}$ of this type from other possible competitor models of New Physics by looking at its possible combined effects in the hadronic channels at LEP2.

III) In order to perform a reasonably simple search of the aforementioned effects, a certain reduction of the number of $Z^{\prime}$ parameters must be enforced. This can be achieved either by imposing a number of purely phenomenological restrictions or by selecting a specific model. We shall immediately illustrate possible ways of carrying on this program in what follows.

A first simple way to eliminate one of the four quark parameters $\xi_{i}$ is that of using the available experimental information on the $Z$ hadronic width $\Gamma_{h}$. In our analysis, any possible deviation of a given observable from the corresponding SM prediction is interpreted as a " $Z$ ' shift". For $\Gamma_{h}$ the most recent analyses [3] give:

$$
\frac{\Gamma_{h}^{(e x p)}-\Gamma_{h}^{(S M)}}{\Gamma_{h}^{(S M)}}=+0.0005 \pm 0.0014 .
$$

In the situation that we are considering, the l.h.s. of eq. (5) can also be written as $\delta \Gamma_{h}^{\left(Z^{\prime}\right)} / \Gamma_{h}$. For this quantity one can write, neglecting for simplicity terms of higher order in "small" parameters (like the $Z-Z^{\prime}$ mixing angle $\vartheta_{M}$, defined in the conventional way):

$$
\frac{\delta \Gamma_{h}^{\left(Z^{\prime}\right)}}{\Gamma_{h}} \simeq \frac{3}{2} \delta_{\rho}^{\left(Z^{\prime}\right)}+\frac{36}{59} \vartheta_{M}\left[\xi_{A u}+4 g_{v u}^{2} \xi_{V u}+\frac{3}{2} \xi_{A d}+6 g_{v d}^{2} \xi_{V d}\right]
$$

where, for reasonably large $Z^{\prime}$ masses:

$$
\delta_{\rho}^{\left(Z^{\prime}\right)} \simeq \vartheta_{M}^{2} \frac{M_{Z^{\prime}}^{2}}{M_{Z}^{2}}
$$

Imposing the equality of eqs. (5) and (6) gives rise to a linear relationship between the four parameters $\xi_{i}$, that reads:

$$
\xi_{A u}=-\frac{3}{2} \xi_{A d}-6 g_{v d}^{2} \xi_{V d}-4 g_{v u}^{2} \xi_{V u} \pm \delta
$$


where $\delta$ depends on $M_{Z^{\prime}}, \delta_{\rho}^{\left(Z^{\prime}\right)}$ and the experimental result in the r.h.s. of eq. (5). Assuming for $\delta_{\rho}^{\left(Z^{\prime}\right)}$ the reasonable bound [13]:

$$
\delta_{\rho}^{\left(Z^{\prime}\right)} \lesssim 3 \times 10^{-3}
$$

one sees that the contribution to $\delta$ of the experimental input $\sim\left(\Gamma_{h}^{(e x p)}-\Gamma_{h}^{(S M)}\right)$ can be essentially ignored in the region of large $\left|\xi_{A u}\right|$ in which we are interested, so that one can write for practical purposes:

$$
\delta \simeq \frac{5 M_{Z^{\prime}}}{2 M_{Z}} \sqrt{\delta_{\rho}^{\left(Z^{\prime}\right)}} \simeq 27\left(\frac{M_{Z^{\prime}}}{1 T e V}\right) \sqrt{\delta_{\rho}^{\left(Z^{\prime}\right)}}
$$

The previous quantity is such that, in the region of interest for LEP2, $M_{Z^{\prime}} \leq 1 \mathrm{TeV}$, and for the worst assumption on $\delta_{\rho}^{\left(Z^{\prime}\right)}$,

$$
|\delta| \lesssim 1
$$

A shift like that of eq. (10) would therefore, at most, change the value of $\xi_{A u}$ (for fixed $\left.\xi_{V u}, \xi_{A d}, \xi_{V d}\right)$ by an amount sensibly smaller than the "relevant" $\left(\left|\xi_{A u}\right| \gtrsim 3\right)$ ones. In practice, and for simplicity reasons, we shall incorporate from now on the parameter $\delta$ of eq. (8) into a generalized definition of $\xi_{A u}$, that will read:

$$
\xi_{A u}^{\prime} \equiv \xi_{A u} \pm \delta \equiv-\frac{3}{2} \xi_{A d}-6 g_{v d}^{2} \xi_{V d}-4 g_{v u}^{2} \xi_{V u}
$$

and would be practically identical with the conventional $\xi_{A u}$ in the "large" $\xi_{A u}$ region for $M_{Z^{\prime}}$ sufficiently below one $\mathrm{TeV}$ or $\delta_{\rho}^{\left(Z^{\prime}\right)}$ sufficiently smaller than the bound of eq.(9).

As a second constraint to be used in our analysis, we shall use the condition that can be derived from the request (that we shall maintain in this paper) that the considered $Z^{\prime}$ is "reasonably" narrow. In practice, we shall make this statement more quantitative by demanding that

$$
\frac{\Gamma_{Z^{\prime}}}{M_{Z^{\prime}}} \lesssim 0.20
$$

From the expression of the $Z^{\prime}$ width given e.g. in Ref. [1] one sees that eq. (13) leads to the constraint

$$
\frac{3 \alpha}{4 s_{e f f}^{2} c_{e f f}^{2}}\left[\frac{1}{4}\left(\xi_{A u}^{2}+\xi_{A d}^{2}\right)+g_{V u}^{2} \xi_{V u}^{2}+g_{V d}^{2} \xi_{V d}^{2}\right] \lesssim 0.20
$$

that becomes numerically $\left(s_{\text {eff }}^{2}=1-c_{e f f}^{2}=0.232\right)$

$$
\left[\xi_{A u}^{2}+\xi_{A d}^{2}+0.48 \xi_{V d}^{2}+0.15 \xi_{V u}^{2}\right] \lessgtr 26
$$

Eqs. (8) and (15) are the two purely phenomenological constraints that we shall use in our analysis. To further reduce the number of parameters, extra theoretical assumptions must be enforced. One natural possibility, that has been suggested in Ref. [2], is that of assuming that the $Z^{\prime}$ is associated to an extra $U(1)$ that commutes with the standard 
$S U(2)_{L} \otimes U(1)_{Y}$ group. In terms of the $Z^{\prime}$ quark effective couplings, this means that $g_{u L}^{\prime}=g_{d L}^{\prime}$ and therefore that

$$
g_{v u} \xi_{V u}+\frac{1}{2} \xi_{A u}=g_{v d} \xi_{V d}-\frac{1}{2} \xi_{A d} .
$$

Following the suggestion of Ref. [2], and assuming that anomalies can be cancelled by a proper ad hoc mechanism that is beyond the purposes of this analysis, we shall now investigate the visible properties at LEP2 of a model that meets the constraints eqs. (8), (15) and (16) and that we shall call from now on model $A$. Briefly, we shall perform the following operations:

I) we shall concentrate on the experimental variables $\sigma_{b}=\sigma\left(e^{+} e^{-} \rightarrow b \bar{b}\right), A_{F B, b}$ (the forward-backward asymmetry for $b \bar{b}$ production) and $\sigma_{5}$ (the cross section for hadron production at LEP2). We shall work assuming a total c.m. energy of $175 \mathrm{GeV}$ and a total integrated luminosity of $500 \mathrm{pb}^{-1}$, following the discussion on the expected experimental accuracies given in Ref. [10]. In particular, the relative experimental errors on $\sigma_{5}, \sigma_{b}$ and $A_{F B, b}$ will be taken as $0.7 \%, 2.5 \%$ and $5 \%$, respectively.

II) The $Z^{\prime}$ parameters will be chosen in the following way: the two $b$ effective couplings $\xi_{V b}, \xi_{A b}$ will be left free to vary, within the allowed region limited by the bound imposed by the request of small $Z^{\prime}$ width, eq. (15). The two $u$ couplings $\xi_{A u}, \xi_{V u}$ will be fixed by the conditions of eqs. (8) and (16). In practice this sets a limitation on $\left|\xi_{i}\right|$ of the kind $\left|\xi_{i}\right| \lesssim$ $3-4$. We shall neglect for a first investigation the extra parameter $\delta$, remembering from our previous discussion that values of $\delta$ below the limit $|\delta| \lesssim 1$ will not change the relevant conclusions (as we checked for intermediate $\delta$ values) but will be simply reabsorbed into a redefinition of an effective $\xi_{A u}^{\prime}$ parameter, eq. (12). For purely orientative reasons, $M_{Z^{\prime}}$ has been fixed at a value $M_{Z^{\prime}}=800 \mathrm{GeV}$ that was suggested by our analysis of the CDF events [1]]; rescaling the results to different $M_{Z^{\prime}}$ values is trivial as suggested by eqs. (2) $-(4)$.

III) The two $Z^{\prime}$ lepton parameters $g_{V l}^{\prime}, g_{A l}^{\prime}$ have been analyzed in the following way. We have set

$$
r=\frac{g_{A l}^{\prime}}{g_{V l}^{\prime}}
$$

and considered different cases at fixed $r$. In particular, we have analysed the special situations $|r| \gg 1$ (predominant axial $Z^{\prime}$ lepton coupling), $r=0$ (predominant vector coupling), $r= \pm 1$ (left and right-handed couplings); these cases should give a picture of various possibilities that might reasonably occur in a class of theoretical models.

IV) Once the values of $r$ and of $M_{Z^{\prime}}$ are fixed, the three experimental quantities that we have considered only depend on two residual effective parameters $\sim g_{V l}^{\prime} \cdot \xi_{V b}, g_{V l}^{\prime} \cdot \xi_{A b}$. This means that the three relative $Z^{\prime}$ shifts on the observables will be related by a certain relationship that will not depend on the previous residual parameters, and will therefore lie on a certain $3 \mathrm{~d}$ region fully characteristic of the $Z^{\prime}$ model. Following the notation of a previous work [14], we have called " $Z$ ' reservation" this region and have computed it for the various $r$ values. The aim of this operation was also that of showing that certain possible competitor theoretical models will generate different "reservations", which will be shown at the end of this paper. 


\section{Inclusion of QED corrections}

The practical evaluation of the $Z^{\prime}$ shift must necessarily take into account the potentially dangerous QED radiation effects. In order to accomplish this goal, we have upgraded a Monte Carlo program, previously used for an analysis of the $Z^{\prime}$ effects on the leptonic observables at LEP2 [14], to give predictions for the relevant hadronic observables $\sigma_{b}$, $A_{F B, b}$ and $\sigma_{5}$, keeping under control the bulk of the contribution due to the emission of soft and collinear (undetected) initial-state photonic radiation. In order to assess the normalization of the $Z^{\prime}$ shifts as returned by the program, the predictions for the hadronic SM quantities have been compared with the high-precision theoretical results of typically used LEP software [15] and found to be in agreement at the level of few per cent for the integrated cross sections and within $1 \%$ for the $b$-asymmetry. However, these small discrepancies, which have to be essentially ascribed to neglecting final-state QCD corrections, mixed electroweak-QCD contributions and finite-mass effects in our calculation, marginally affect the relevant conclusions because the neglected short-distance corrections largely cancel out in the numerical evaluation of the relative shifts in which we are interested.

Following the strategy already employed in Ref. [14], the $Z^{\prime}$ contribution has been included in the lowest-order calculation of the cross section implemented in the Monte Carlo computing the $s$-channel amplitudes associated to the production of a $q \bar{q}$ (massless) pair in $e^{+} e^{-}$annihilations mediated by the exchange of a photon, a standard $Z$ and an additional $Z^{\prime}$ boson. The resulting cross section has been dressed with leading weak corrections included in the form of Improved Born Approximation and the potentially dangerous distortions introduced by initial-state QED radiation have been kept under control employing the QED structure function approach [16], i.e. convoluting the shortdistance cross section $\sigma_{0}$ with electron(positron) structure functions as follows \&

$$
\sigma\left(q^{2}\right)=\int d x_{1} d x_{2} D\left(x_{1}, q^{2}\right) D\left(x_{2}, q^{2}\right) \sigma_{0}\left(x_{1} x_{2} q^{2}\right) \Theta(\text { cuts })
$$

where $D\left(x, q^{2}\right)$ is the electron (positron) structure function (whose typical expression can be found elsewhere [16, 17]) and $\Theta$ (cuts) stands for the rejection algorithm to implement possible experimental cuts. In our analysis, we use the cut $s^{\prime} / q^{2}>0.35$ (where $s^{\prime}=x_{1} x_{2} q^{2}$ is the invariant mass of the event after initial-state radiation) imposed in order to avoid, according to standard LEP2 selection criteria, the unwanted events due to $Z$ radiative return and hence disentangle the interesting virtual $Z^{\prime}$ effects.

The numerical results obtained via the Monte Carlo program have been compared with the analytical formulae given in eqs. (2)-(4), in order to estimate the size of those Born level contributions neglected in the derivation of the analytical expressions for the $Z^{\prime}$ shifts written in those equations and taken into account in the numerical simulation. For simplicity reasons, this comparison has been performed assuming the relations among

\footnotetext{
${ }^{2}$ The actual implementation of QED corrections is performed, in the Monte Carlo code, at the level of the differential cross section, taking into account all the relevant kinematical effects according e.g. to Ref. [17]; in the present paper only a simplified formula is described, for the sake of simplicity.
} 
the couplings valid for model $A$ but neglecting the QED corrections. The content of this analysis is summarized in Fig. 1 for the illustrative case $r=-1$. In this plot one can see: the results for the three hadronic observables as predicted by the analytical formulae of eqs. (2) -(4) (Fig. 1a); the numerical results obtained with the Monte Carlo code, switching off both the contribution of the $Z^{\prime}$-exchange squared amplitude and the $Z^{\prime}$ width, the latter calculated according to the expression given in Ref. [1] (Fig. 1b); the numerical results obtained with the Monte Carlo, switching off the contribution of the $Z^{\prime}$ width but retaining the $Z^{\prime}$ squared amplitude (Fig. 1c); the full numerical results of the Monte Carlo, with both the $Z^{\prime}$ squared amplitude and $Z^{\prime}$ width switched on (Fig. 1d). As can be seen from the comparison between Fig. 1a and Fig. 1b, there is a rather satisfactory agreement between analytical and Monte Carlo numerical estimates when the contributions due to non-linear effects in the $Z^{\prime}$ (lepton and quark) couplings introduced in the calculation of the relative deviations by the $Z^{\prime}$ squared amplitude and the $Z^{\prime}$ width are discarded. On the other hand, this step by step investigation also points out that, in view of a more precise evaluation of the global $Z^{\prime}$ virtual effects, the non-linear contributions in the $Z^{\prime}$ couplings have to be accounted for, whereas they can be neglected if one is interested in the order of magnitude of the relevant shifts. These general conclusions remain valid for all the values of $r$ that we have considered.

\section{Numerical results and discussion}

After this preliminary check of our computational algorithm, we have drawn, for all the representative values of $r$, the characteristic surfaces of model $A$ in the $3 \mathrm{~d}$-space of the shifts in the three considered hadronic channel observables. Contrary to the previous preliminary investigation, we have now fully imposed both phenomenological conditions eq. (12), (15) and eq. (16) and the effect due to QED radiation. Moreover, we have been limited in our analysis to values of the leptonic couplings that are essentially "small". In particular, we have assumed that these couplings would not produce any observable effect at LEP2 in the leptonic channels. From the previous analysis performed in Ref. [8]

this means that, to $95 \% \mathrm{CL}$, the two $Z^{\prime}$ couplings $g_{V l}^{\prime}, g_{A l}^{\prime}$ will have to lie within a certain region (that depends on the fixed $M_{Z}^{\prime}$ value) that corresponds to an ellipse in their plane. We shall refer to this region as to the "leptonic channel ellipse" at LEP2.

The results of our calculations for different $r$ are shown in Figs. 2-6. The central box in the Figures corresponds to a "black" region of non visibility, drawn for simplicity in a rather conservative way starting from the assumed experimental accuracies (a more accurate estimate of the "black" region would be straightforward, but would not change appreciably our discussion). Should the experimental point lie on one of the various reservations, outside the non observability region, it would be a reasonable indication of the existence of a $Z^{\prime}$ of the considered type.

Two more things should be now stressed. The first one is that the observability domains in the various cases are evidently not empty, as one sees from inspection. This means that for all the relevant values of $r$, and for $Z^{\prime}$ leptonic couplings not visible in the 
LEP2 leptonic channels, there are values of the $Z^{\prime}$ quark couplings in model A, consistent with the available experimental information eq. (5), that would make a narrow $Z^{\prime}$ visible in the LEP2 hadronic channels. The second one is that our $3 \mathrm{~d}-$-analysis might be able in the considered example to differentiate genuine $Z^{\prime}$ effects from effects due to competitor theoretical models, owing to the particularly simple parameterization that is valid for the representative model $A$.

To make this statement more quantitative, we have considered the case of a model where anomalous triple gauge couplings [18] are present. We have followed the notations and the assumptions of Hagiwara et al. [19], and worked in the framework of Refs. [11], [12], where it has been shown that the number of relevant parameters of the model in the final two fermion channel at LEP2 is reduced to two [20]. For this model, that we shall call model $B$ from now on, it will be therefore possible to draw, in the same $3 \mathrm{~d}$ space of Figs. 2-6, an analogous surface that will be its characteristic feature. We have computed this curve only in Born approximation, where it can be given a simple analytic expression (all details can be easily derived from a generalization of the analysis performed in Ref. [20]). A more accurate determination would be possible, but we know from our previous discussion that at least the dominant shape of the surface would not change. The resulting domain is shown in Fig. 7, and one can see from inspection that it is different from the domains allowed for model A with the considered $r$ values. There would be thus a clean differentiation of model $\mathrm{A}$, from at least one reasonable alternative competitor model, provided by a careful analysis of the LEP2 hadronic channels.

The final point that we want to discuss now is the one that was raised in the introduction of our paper. Using the same computational algorithm that led us to the previous conclusions, we have tried to determine, for a convenient choice of the enhanced $Z^{\prime}$ quark couplings, the minimum $Z^{\prime}$ lepton couplings that would produce a visible effect in the hadronic channels of LEP2. For practical reasons we have now focused our attention on the two hadronic cross sections $\sigma_{5}$ and $\sigma_{b}$, and imposed the visibility condition at two sigmas for each observable, given the assumed experimental precision.

In principle, our analysis could be performed allowing the $Z^{\prime}$ quark couplings to vary in a proper domain where the three conditions eqs. (12), (13) and (16) are satisfied. This would then define a real set of minimal values for $g_{V l}^{\prime}, g_{A l}^{\prime}$. But for the first purposes of indication we thought that it was enough to consider a couple of typical sets of $Z^{\prime}$ quark couplings that met all the imposed requests and represented, so to say, two completely reasonable examples. In this spirit, we have chosen the two representative couples $\xi_{V b}=$ $\xi_{A b}=2$ (couple I) and $\xi_{V b}=\xi_{A b}=-2$ (couple II); the remaining $Z^{\prime} u u$ couplings have been fixed by the conditions eqs. (12) and (16), neglecting for simplicity the small parameter $\delta$. With these choices, the constraint eq. (13) is met and all the values of the $Z^{\prime}$ quark couplings lie in a reasonable range, that still corresponds to a typical electroweak strength, as discussed in Ref. [1].

The results of our investigation are shown in Fig. 8, in correspondence to the special choice $M_{Z}^{\prime}=800 \mathrm{GeV}$. The main features, in our opinion, are the following ones:

I) the role of $\sigma_{b}$ and $\sigma_{5}$ is, in this search, complementary: the first observable is more reactive to small $Z^{\prime}$ axial lepton couplings, the second one to small vector ones. From the 
combination of the two measurements, a region of $\left(g_{V l}^{\prime}, g_{A l}^{\prime}\right)$ values of modulus typically around $\sim 0.1-0.2$ appears to be still able to produce visible effects;

II) typical values of the $Z^{\prime}$ lepton couplings that would make the $Z^{\prime}$ visible in the final hadronic channels are much smaller than typical values that would not make it visible in the final lepton channels (inside the lepton ellipse depicted in Fig. 8).

These main indications that can be derived from an inspection of Fig. 8 can be generalized to a more specific search, where a proper minimization program has been used. The results of this calculation, that has been performed in Born approximation for the reasons that we have already discussed, indicate that the minimum size of the $Z^{\prime}$ lepton couplings in model $A$ that would still be seen in the combined analysis of $\sigma_{b}$ and $\sigma_{5}$ at LEP2 (for values of the $Z^{\prime}$ quark couplings satisfying our requests) are, for a general $Z^{\prime}$ mass:

$$
\begin{aligned}
& \left|g_{V l}^{\prime}\right| \gtrsim 0.1\left[\frac{M_{Z^{\prime}}}{1 T e V}\right] \\
& \left|g_{A l}^{\prime}\right| \gtrsim 0.1\left[\frac{M_{Z^{\prime}}}{1 T e V}\right]
\end{aligned}
$$

that are approximately five times smaller than the limits from the lepton ellipse derived from the lepton channels at LEP2.

Our results eqs. (19), (20) should now be compared with the available experimental information from LEP1/SLC. This can be done if an analysis of the latter information is performed like in Ref. [1], i.e. allowing the $Z^{\prime}$ couplings to be completely free (but still family independent as in our assumption). This leaves six parameters in charged leptonquark sector, to be analyzed together with the extra parameters $\vartheta_{M}$ (the usual mixing angle) and $\delta \rho^{\left(Z^{\prime}\right)}$, the modification of the $\rho$ parameter due to a $Z^{\prime}$, whose relationship with $\vartheta_{M}$ is given in eq. (7). Assuming a bound for $\delta \rho^{\left(Z^{\prime}\right)}$ like that of eq. (9), i.e. $\sim 3 \times 10^{-3}$, one obtains therefore an upper bound for the mixing angle:

$$
\left|\vartheta_{M}\right| \lesssim \sqrt{0.003} \frac{M_{Z}}{M_{Z^{\prime}}}
$$

From the available LEP1/SLC data one can derive, in general, bounds for the product of $\vartheta_{M}$ with $Z^{\prime}$ couplings. Assuming that $\vartheta_{M}$ saturates its bound, eq. (21) will consequently provide the minimal bounds of lepton couplings. From the last communicated data [3] where both LEP1 and SLC data were used, one would derive at one standard deviation and for the bound on $\delta \rho^{\left(Z^{\prime}\right)}$ of eq. (9):

$$
\left|g_{V l}^{\prime}\right| \lesssim 0.4\left(\frac{M_{Z^{\prime}}}{1 T e V}\right) \quad ; \quad\left|g_{A l}^{\prime}\right| \lesssim 0.5\left(\frac{M_{Z^{\prime}}}{1 T e V}\right)
$$

Note that, if a value of $\delta \rho^{\left(Z^{\prime}\right)}$ as low as one permille were assumed, the corresponding minimal values would increase by almost a factor of two.

When one repeats, under the same previous assumptions, this analysis for the quark couplings, the corresponding minimal bands are in fact much larger, and somehow depending on which set of data (LEP1 or SLC) is used. Using, whenever possible, the 
average LEP/SLC data and following the same procedure as in Appendix A of Ref.[四], we have found the following results:

$$
\begin{gathered}
\eta_{M} \xi_{V b} \simeq(-9.99 \pm 14.87)\left(\frac{M_{Z^{\prime}}}{1 T e V}\right) \\
\eta_{M} \xi_{A b} \simeq(+5.86 \pm 7.17)\left(\frac{M_{Z^{\prime}}}{1 T e V}\right) \\
\eta_{M} \xi_{V c} \simeq(+0.028 \pm 22.77)\left(\frac{M_{Z^{\prime}}}{1 T e V}\right) \\
\eta_{M} \xi_{A c} \simeq(-0.74 \pm 7.28)\left(\frac{M_{Z^{\prime}}}{1 T e V}\right)
\end{gathered}
$$

where $\eta_{M}= \pm 1$. As one sees, for the four effective ratios $\xi_{i}$, values of order $\sim 10\left[M_{Z^{\prime}} / 1 \mathrm{TeV}\right]$ are still perfectly consistent with the available experimental information. Enforcing the further constraint of reproducing a possible excess of dijet events at CDF [4] would select values of $\left|\xi_{i}\right|$ of about 3-4 as shown in Ref. [1]. One sees therefore that the values of $\xi_{i}$ that we used in the present paper are perfectly consistent with all available data.

\section{Conclusions}

In conclusion, we have analyzed a rather general theoretical model where one $Z^{\prime}$ exists, related to an extra $U(1)$ that commutes with the Standard electroweak group, as suggested in Ref. [2]. Imposing on the $Z^{\prime}$ quark couplings to satisfy the single phenomenological condition dictated by the available experimental value of the $Z$ hadronic width measured at LEP1, and assuming that the $Z^{\prime}$ width is reasonably narrow, we have taken into account a range of $Z^{\prime}$ quark couplings that are larger than the corresponding ones for the SM $Z$, but perfectly consistent with all the available experimental information.

In such a picture, we have verified that the minimum values of the $Z^{\prime}$ lepton couplings that would still give visible effects in the two hadronic cross sections $\left(\sigma_{b}\right.$ and $\left.\sigma_{5}\right)$ measurable at LEP2 are those of eqs. (19) and (20), at least five times smaller than the existing bounds from LEP1/SLC (and also five times smaller than the future bounds from negative searches in the final lepton channels at LEP2). For values of the $Z^{\prime}$ lepton couplings $g_{V, A l}^{\prime}$ larger than those of eqs. (19), (20) we have shown that the effects of the considered model at LEP2 would be seen in the final hadronic channels in a "reasonably" unambiguous way, for all possible choices of the ratio $g_{V l}^{\prime} / g_{A l}^{\prime}$. We believe thus to have somehow quantified the typical values of $\left|g_{V l}^{\prime}\right|,\left|g_{A l}^{\prime}\right|$ below which it is allowed to identify a "small" $Z^{\prime}$ lepton coupling with a "vanishing" one. For values of the $\left|g_{l}^{\prime}\right|$ moduli beyond the $\sim 0.1\left[M_{Z}^{\prime} / 1 T e V\right]$ threshold, the previous identification might lead to very negative consequences.

\section{Acknowledgements}

We would like to thank O. Nicrosini and A. Rotondi for useful discussions. 


\section{References}

[1] P. Chiappetta, J. Layssac, F.M. Renard and C. Verzegnassi, Phys. Rev. D54 (1996) 789.

[2] G. Altarelli, N. Di Bartolomeo, F. Feruglio, R. Gatto and M. L. Mangano, Phys. Lett. B375 (1996) 292 .

[3] For the last recent situation see the talks of A. Blondel and M.W. Grünewald at the Warsaw conference on High Energy Physics (1996).

[4] E. Buckley-Geer, FERMILAB-Conf-95/316-E (1995), to appear in Proceedings of EPS Conference on HEP, Brussels.

[5] See the discussion given in Physics at LEP2, CERN Report 96-01, G. Altarelli, T. Sjöstrand and F. Zwirner Eds.

[6] K.S. Babu, C. Kolda and J. March-Russell, hep-ph/9603212, Phys. Rev. D, to appear.

[7] A.E. Faraggi and M. Masip, hep-ph/9604302 and references therein.

[8] P. Chiappetta et al., in Physics at LEP2, CERN Report 96-01, G. Altarelli, T. Sjöstrand and F. Zwirner Eds., Vol. 1, p. 577.

See also:

A. Leike and S. Riemann, DESY 96-111, LMU-02/96, hep-ph/9607306.

[9] A. Djouadi, A. Leike, T. Riemann, D. Schaile and C. Verzegnassi, Z. Phys. C56 (1992) 289.

[10] See the discussion given in 8 .

[11] F.M. Renard and C. Verzegnassi, Phys. Rev. D52 (1995) 1369.

[12] F.M. Renard and C. Verzegnassi, Phys. Rev. D53 (1996) 1290.

[13] We refer to the analysis presented by P. Langacker, NSF-ITP-95-140, UPR-0683T (1995).

[14] G. Montagna, O. Nicrosini, F. Piccinini, F.M. Renard and C. Verzegnassi, Phys. Lett. B371 (1996) 277 .

[15] G. Montagna, O. Nicrosini, G. Passarino and F. Piccinini, Comput. Phys. Commun. 93 (1996) 120;

G. Montagna, O. Nicrosini, G. Passarino, F. Piccinini and R. Pittau, Comput. Phys. Commun. 76 (1993) 328. 
[16] E. A. Kuraev and V. S. Fadin, Sov. J. Nucl. Phys. 41 (1985) 466;

G. Altarelli and G. Martinelli, Physics at LEP, CERN Report 86-02, J. Ellis and R. Peccei, eds. (Geneva, 1986);

O. Nicrosini and L. Trentadue, Phys. Lett. B196 (1987) 551 ; Z. Phys. C39 (1988) 479.

For a review see also:

O. Nicrosini and L. Trentadue, in Radiative Corrections for $e^{+} e^{-}$Collisions, J. H. Kühn, ed. (Springer, Berlin, 1989), p. 25; in QED Structure Functions, G. Bonvicini, ed., AIP Conf. Proc. No. 201 (AIP, New York, 1990), p. 12.

[17] G. Montagna, O. Nicrosini and F. Piccinini, Phys. Rev D48 (1993) 1021.

[18] K.J.F. Gaemers and G.J. Gounaris, Z. Phys. C1 (1979) 259; K. Hagiwara, R. Peccei, D. Zeppenfeld and K. Hikasa, Nucl. Phys. B282 (1987) 253.

[19] K. Hagiwara, S. Ishihara, R. Szalapski and D. Zeppenfeld, Phys. Lett. B283 (1992) 353 and Phys. Rev. D48 (1993) 2182.

[20] A. Blondel, F.M. Renard, L. Trentadue and C. Verzegnassi, PM/96-14, hep-ph/9605313, to appear in Phys.Rev.D. 


\section{Figure Captions}

Fig. 1 Comparison between analytical formulae and Monte Carlo results for the $Z^{\prime}$ shifts on the hadronic observables. Analytical results according to eqs. (2)-(4) (Fig. 1a); Monte Carlo results without the contributions of the $Z^{\prime}$ squared amplitude and $Z^{\prime}$ width (Fig. 1b); Monte Carlo results without the effect of the $Z^{\prime}$ width but with the contribution of the $Z^{\prime}$ squared amplitude (Fig. 1c); full Monte Carlo results (Fig. 1d).

Fig. $2 Z^{\prime}$ Reservation for model $A$, for $r=-10$.

Fig. 3 The same as Fig. 2 for $r=10$.

Fig. 4 The same as Fig. 2 for $r=-1$.

Fig. 5 The same as Fig. 2 for $r=1$.

Fig. 6 The same as Fig. 2 for $r=0$.

Fig. $7 Z^{\prime}$ Reservation for AGC model (model $B$ ).

Fig. 8 Region for the $Z^{\prime}$ lepton couplings $\left(g_{V l}^{\prime}, g_{A l}^{\prime}\right)$ that would produce a visible $2 \sigma$ effect on $\sigma_{b}$ (Fig. 8a) and on $\sigma_{5}$ (Fig. 8b). The two solid curves correspond to the two representative couples of $\xi$ quark couplings $\xi_{V b}=\xi_{A b}=2$ and $\xi_{V b}=\xi_{A b}=-2$ and are compared with the "leptonic channel ellipse" (dashed curve). QED corrections are included. 


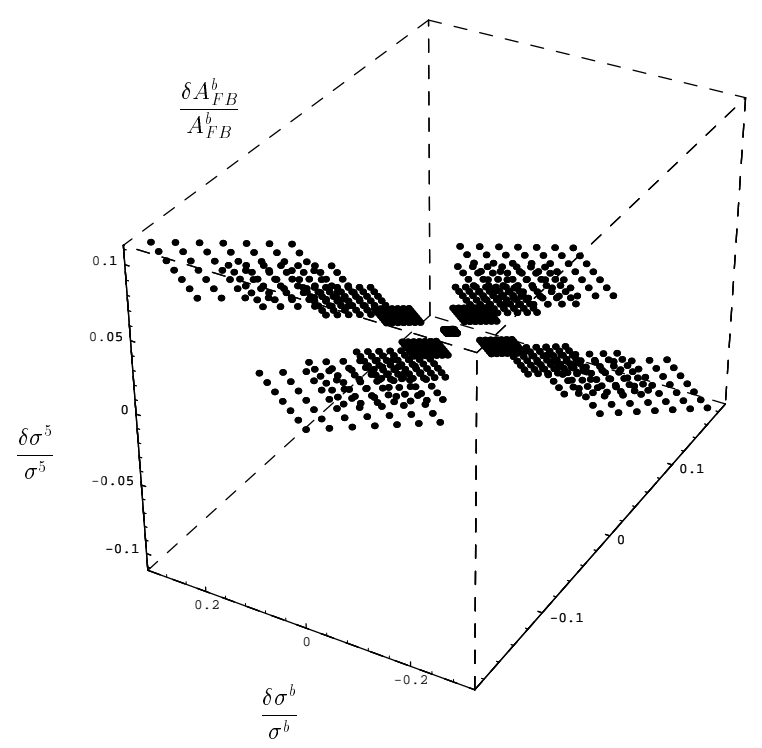

(a)

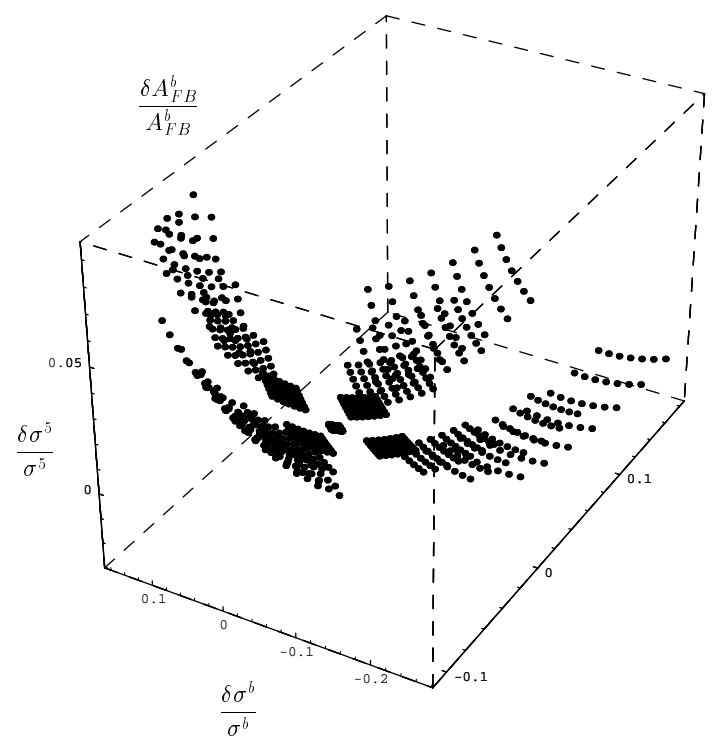

(c)

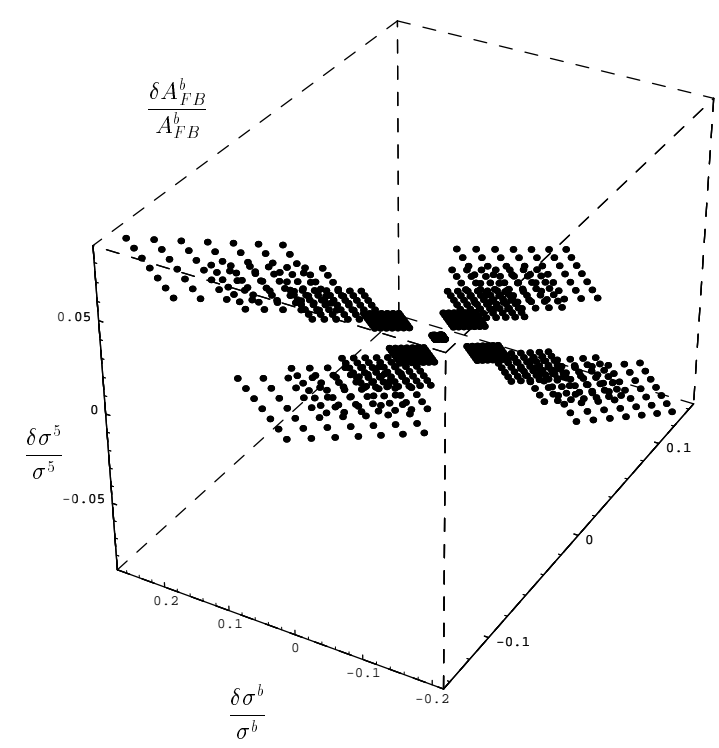

(b)

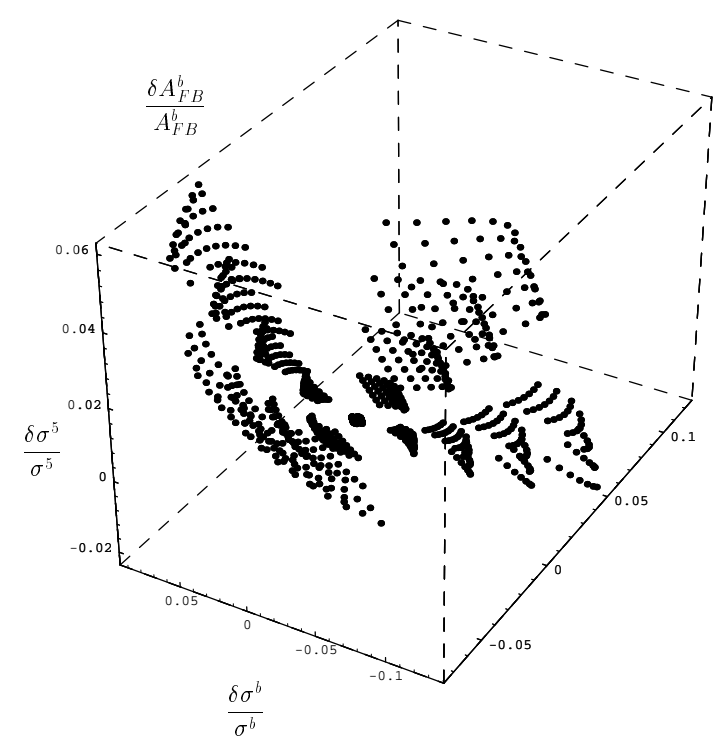

(d)

Fig 1 


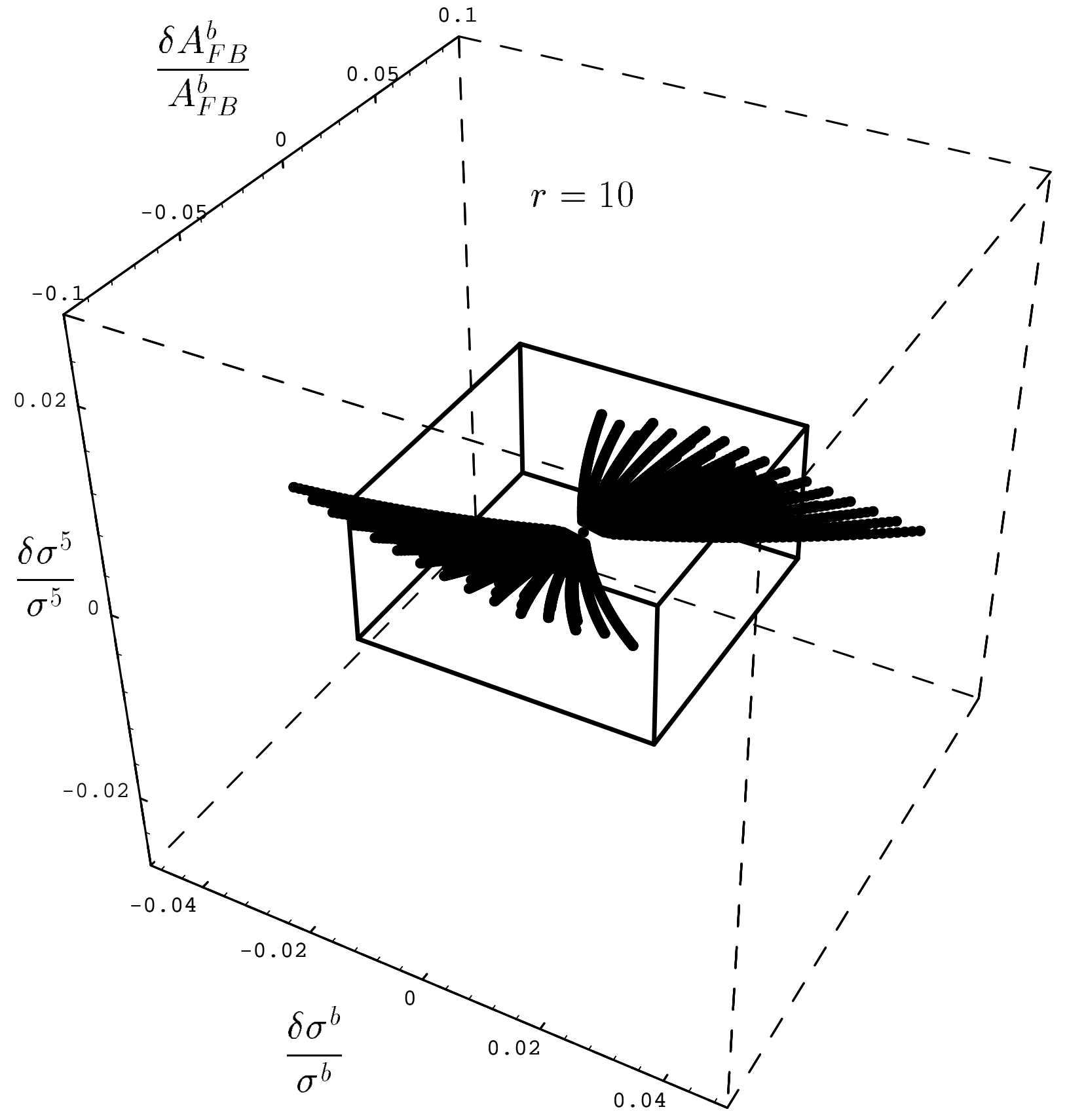

Fig 2 


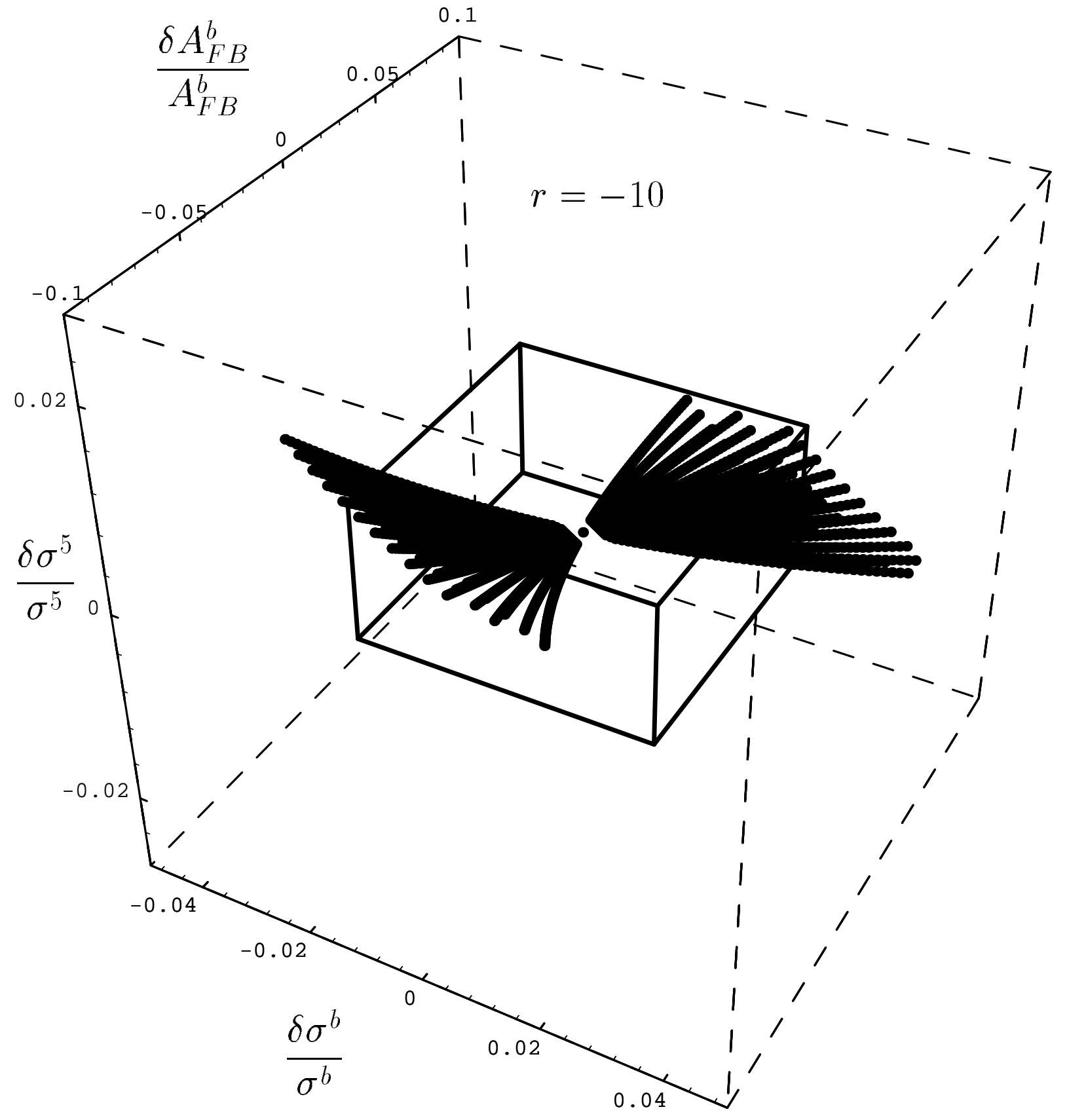

Fig 3 


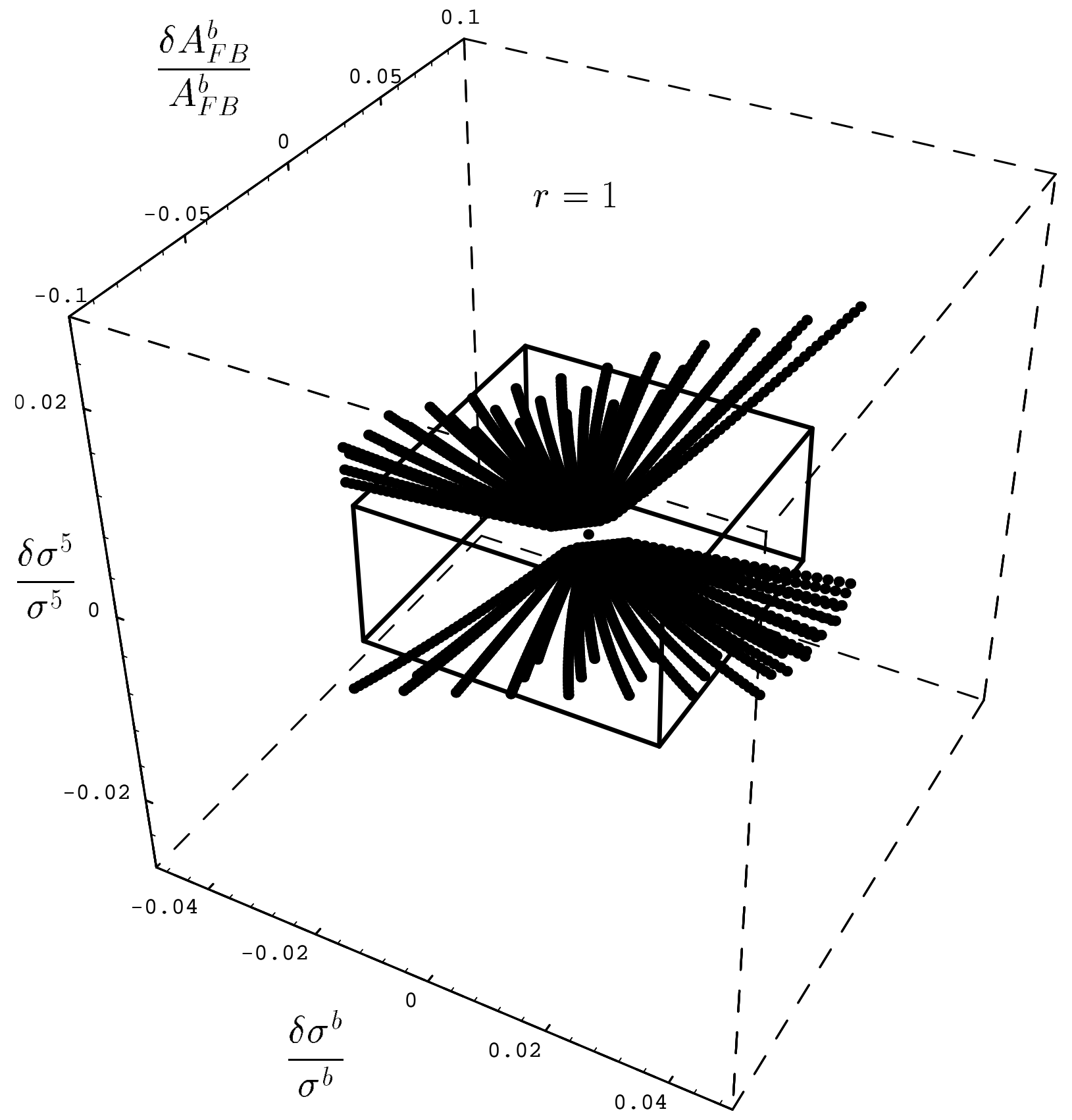

Fig 4 


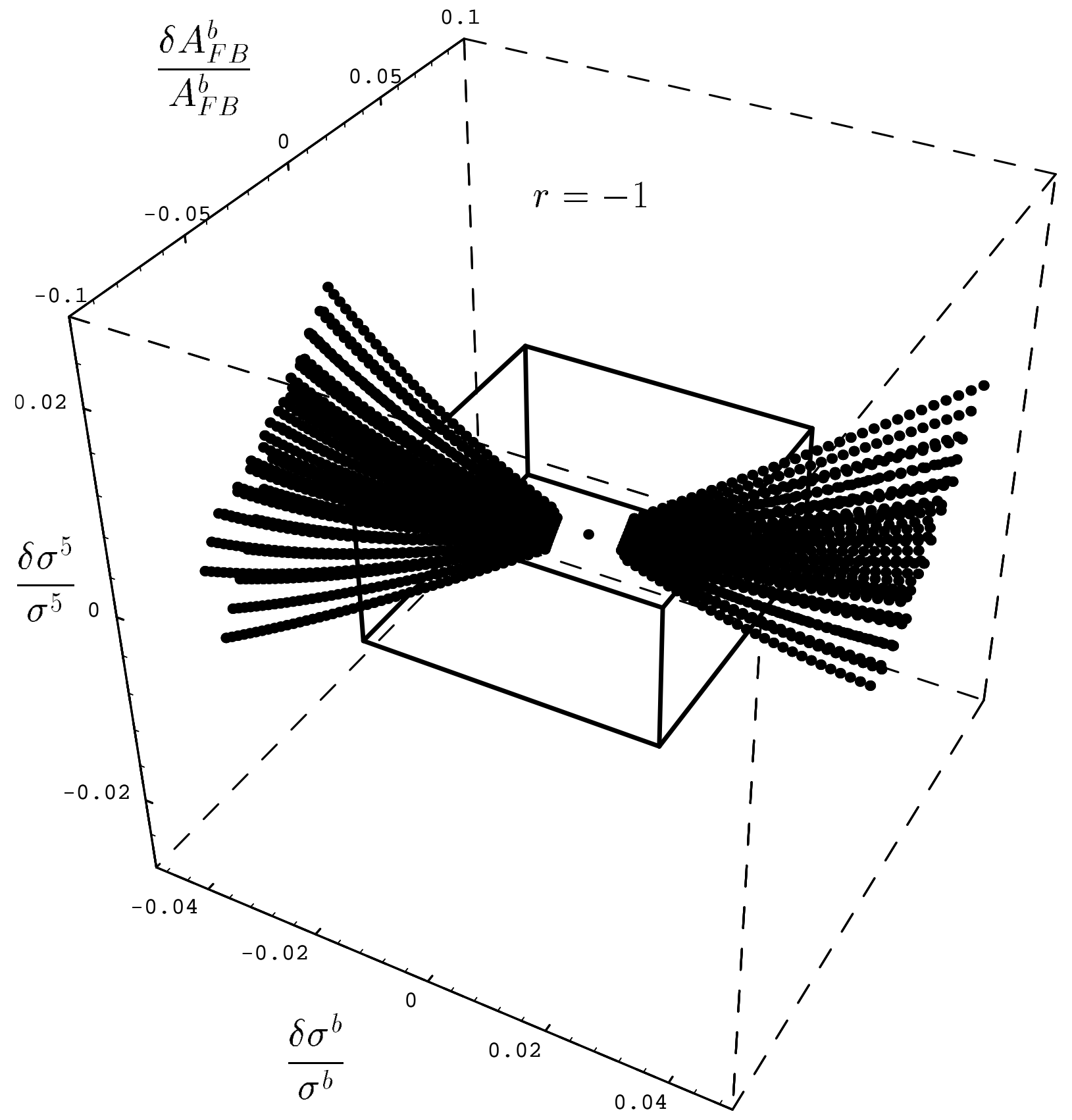

Fig 5 


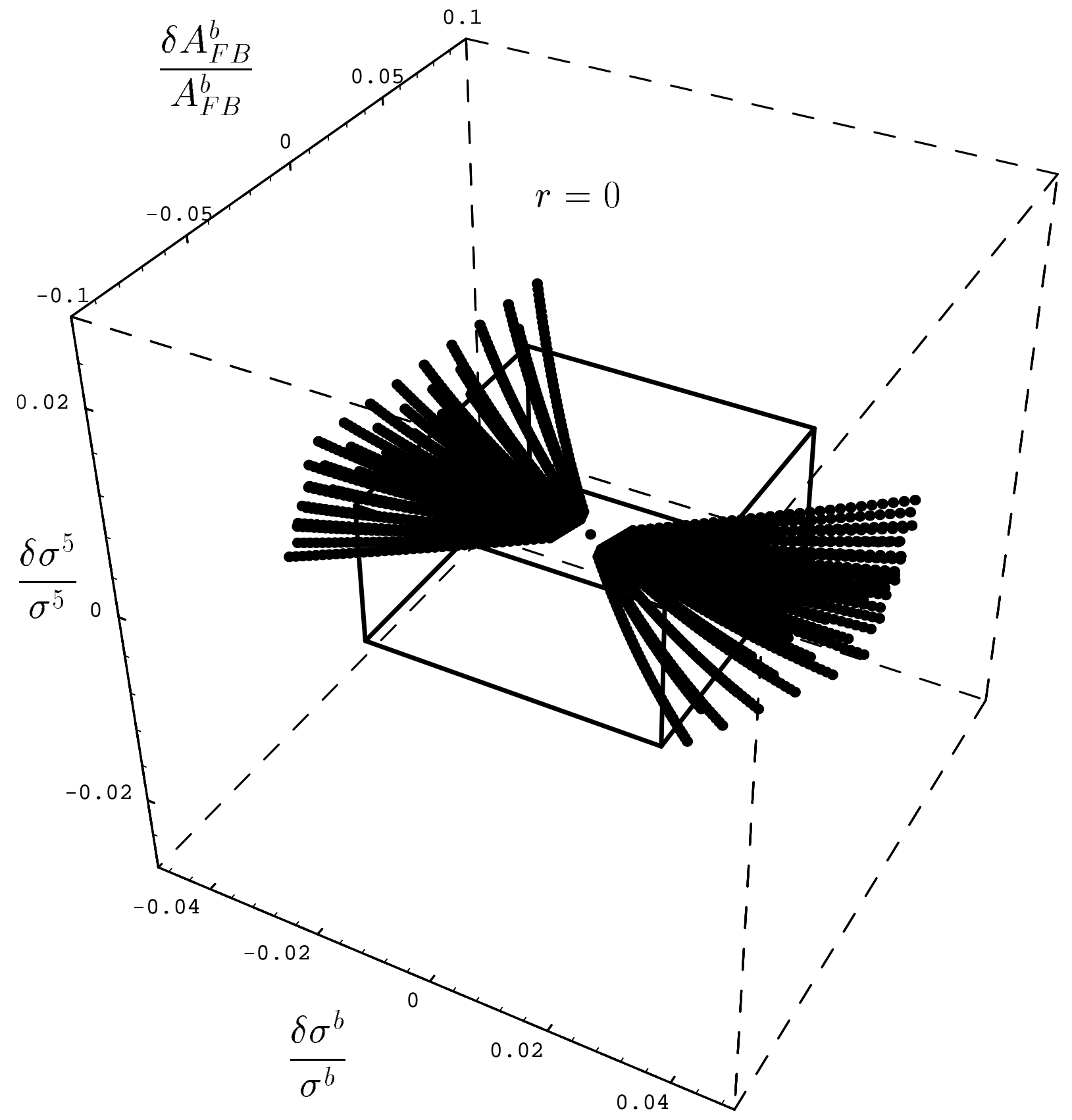

Fig 6 


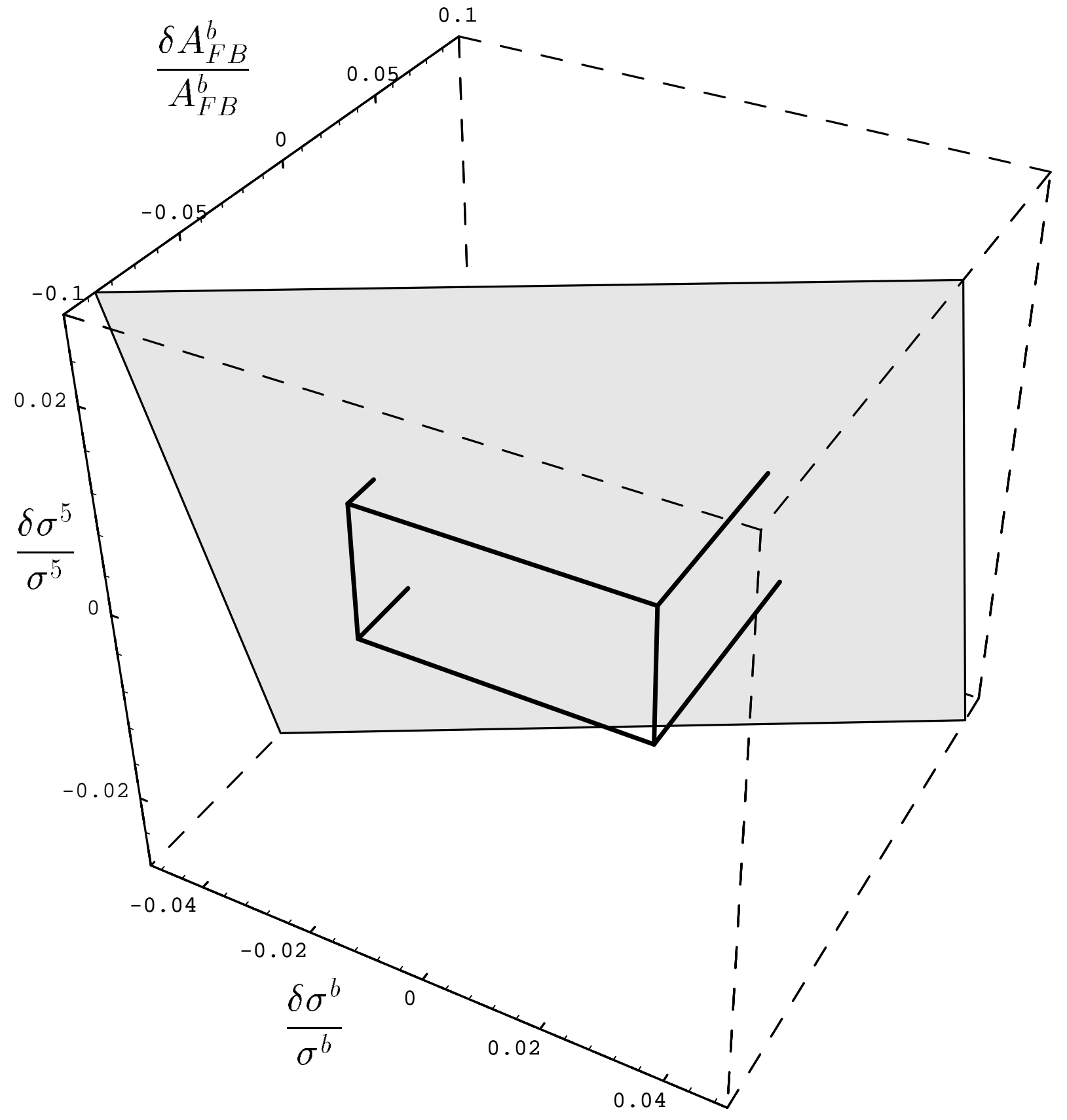

Fig 7 


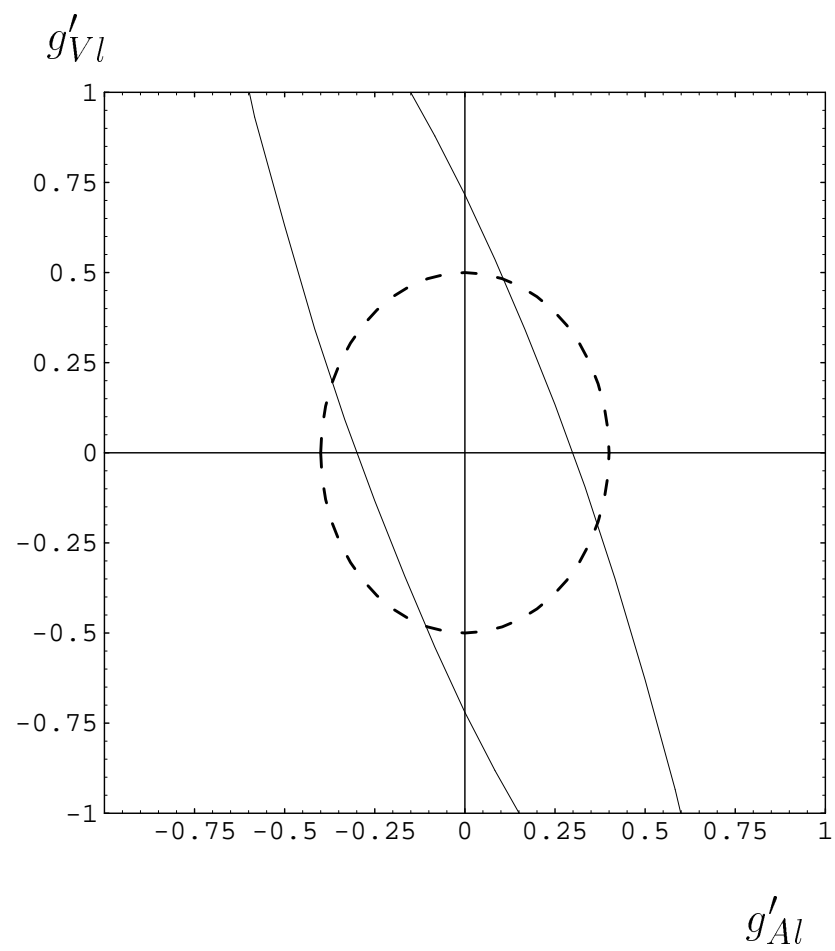

(a)

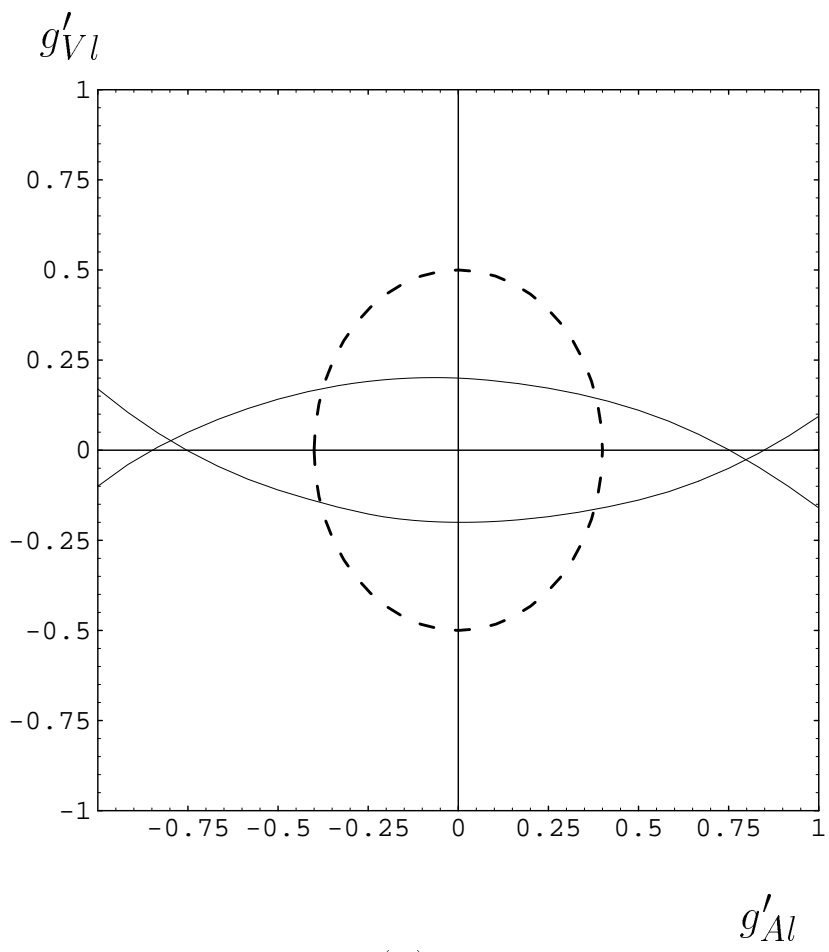

(b)

Fig 8 